\title{
Performance analysis of direction of arrival algorithms for smart antenna
}

\author{
Btissam Boustani, Abdennaceur Baghdad, Aicha Sahel, Abdelmajid Badri \\ Laboratory Electronics, Energy, Automatic and Information Processing, Electrical Engineering Department, \\ Faculty of Science and Technology Mohammedia, Hassan II University Casablanca, Morocco
}

\begin{tabular}{l} 
Article Info \\
\hline Article history: \\
Received Jun 8, 2018 \\
Revised Jun 25, 2019 \\
Accepted Jul 6, 2019 \\
\hline Keywords: \\
DOA algorithm \\
ESPRIT \\
MUSIC \\
MVDR \\
Root-Music \\
Root-WSF \\
Smart antenna system \\
WSF
\end{tabular}

\begin{abstract}
This paper presents the performance analysis of the direction of arrival estimation algorithms such as Estimation of Signal Parameters via Rotational Invariance Technique (ESPRIT), Multiple Signal Classification (MUSIC), Weighted Subspace Fitting (WSF), The Minimum Variance Distortionless Response (MVDR or capon) and beamspace. These algorithms are necessary to overcome the problem of detecting the arrival angles of the received signals in wireless communication. Therefore, these algorithms are evaluated and compared according to several constraints required in smart antenna system parameters, as the number of array elements, number of samples (snapshots), and number of the received signals. The main purpose of this study is to obtain the best estimation of the direction of arrival, which can be perfectly implemented in a smart antenna system. In this context, the ROOTWeighted Subspace Fitting algorithm provides the most accurate detection of arrival angles in each of the proposed scenarios.
\end{abstract}

Copyright $@ 2019$ Institute of Advanced Engineering and Science. All rights reserved.

\section{Corresponding Author:}

Btissam Boustani,

Laboratory Electronics, Energy, Automatic and Information Processing,

Department of Electrical Engineering,

Faculty of Science and Technology Mohammedia,

Hassan II University Casablanca B.P. 146 Mohammedia 20650 Morocco.

Email: btissam.boustani@gmail.com

\section{INTRODUCTION}

Currently, many researchers are interested in new technologies such as the Internet of Things and the 5th generation, to track the excessive growth of wireless technologies. This exponential progress generates increased demand for capacity [1], a need for more radio frequencies [2], excessive appeal for signal processing and space constraints [3], which cannot be satisfied using Conventional antennas, despite the improvements made in the coverage area [3]. Moreover, the use of passive antennas leads to a significant energy wastage, a major interference factor in co-channel, which incites researchers to seek alternative strategies to overcome these encountered problems.

Smart antenna technology is considered as the best solution confronting these problems. It can achieve highly efficient network, maximizing the capacity and improving quality and coverage $[4,5]$. It also referred to adaptive array antennas [6], tends toward creating an adaptive beamforming using a narrow beam in direction of the desired signal while canceling the interference signals. These antennas are used for millimeter waves (mm-wave), Radio Frequency Identification (RFID), Multiple Inputs Multiple Outputs (MIMO), and so on.

Smart antenna system contains two major functions, knowing as the direction of arrival estimation (DOA) and the beamforming given by adaptive algorithms. The classical model of the smart antenna system is considered as showing in Figure 1 [7]. 


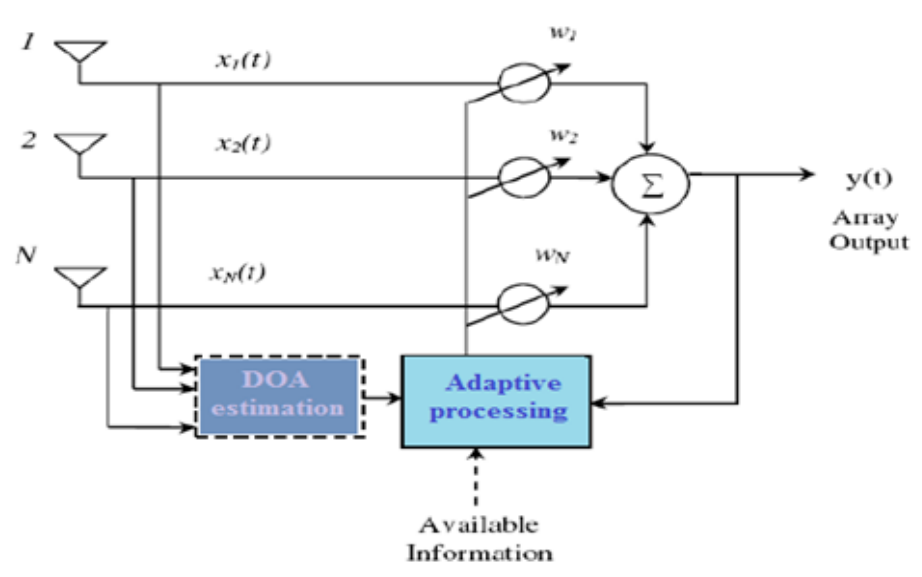

Figure 1. Block diagram of smart antenna system [3]

The direction of arrival (DOA) is one of the most challenging problems for locating and tracking multiple moving sources in different areas, such as radar [8], sonor [9], and mobile communications. The location of sources with distributed sensor arrays can be achieved by estimating the direction of arrival of signals from terminal sources [10, 11].

Various techniques are used to estimate the direction of arrival, among which are high-resolution methods such as Estimation of Signal Parameters via Rotational Invariance Technique (ESPRIT) [12], Multiple Signal Classification (MUSIC) [13], Weighted Subspace Fitting (WSF) [14], The Minimum Variance Distortionless Response (MVDR or capon) [15] and beamspace, these techniques have attracted the most interest and has been the subject of many studies [10-15], however, none of these studies reached the best performance in smart antennas.

In this paper, we focused on one of the main functions, which is the direction of arrival, and we studied all the possibilities of better performance. To do so, we first investigated the algorithms that we worked with, which are beam scan, MUSIC, root-MUSIC, ESPRIT, capon or MVDR, root-WSF. Then we evaluated our system according to the following criterion:

a. The number of array elements

b. The number of samples

c. The number of signals

Finally, we matched the system with the corresponding parameters of smart antenna; in this case, we will work with Wi-Fi parameters as a testing example.

\section{RESEARCH METHOD}

\subsection{DOA estimation}

The main role of the DOA is to estimate the direction of the desired signal by collecting data from the incoming signals received by the antenna array [16]. Adaptive algorithms are the backbone of the smart antenna system. They consist of creating adjustable beams that meet the demands of the system. These algorithms use a set of complex weights to adjust the amplitude or the phase of each elemental antenna of the proposed network. The trained system can only operate by knowing the angles of arrival, where the estimation of the direction of arrival algorithms is then required [17].

\subsection{Conventional DOA}

The conventional DOA beamforming method is also known as the Bartlett method, consist of a power study, in which, over a scan across the angular region of interest, look for the largest output power from the different direction to estimate the desired one [18].

It is also appropriate to proceed to the spatial spectrum method in which the estimation of the direction is made only by corresponding the peak value of the output power with the angle $\Theta$. The expression of the Bartlett method can be given by:

$$
\operatorname{Pconv}(\theta)=\frac{a^{H}(\theta) R_{X X} a(\theta)}{a^{H}(\theta) a(\theta)}
$$


with:

$$
R_{X X}=X(t) X(t)^{H}
$$

In this expression, $\mathrm{a}(\theta)$ and RXX represent respectively the array response vector and the autocovariance matrix of the received signal.

\subsection{MVDR (Capon algorithm)}

Minimum Variance Distortionless Response or Capon beamformer is a solution for the conventional beamformer (the Bartlett method) problem caused when the sources to be located are closer than the beamwidth. When the Bartlett method can't separate them, the MVDR technique interferes to solve the problem [19]. The only adjustment made in the conventional beamformer is the additional matrix inversion $\mathrm{R}_{\mathrm{xx}}$, in which the Capon spatial spectrum is given by:

$$
\operatorname{Pcapon}(\theta)=\frac{1}{a^{H}(\theta) R_{X X}^{-1} a(\theta)}
$$

\subsection{MUSIC algorithm}

The Multiple Signal Classification (MUSIC) algorithm is widely used in adaptive antennas because of the good result estimating the direction of arrival specifically when the signals are uncorrelated and the number of sources is known [20,21]. This method relies on the Eigenstructure of input covariance matrix [21]. Therefore, we chose to work with the signal value decomposition (SVD) technique, of which the covariance matrix is represented as follows:

$$
R=U V U=U_{S} V_{S} U_{S}^{H}+\sigma^{2} U_{n} U_{n}^{H}
$$

$U_{S} V_{S} U_{S}$ And $\sigma^{2} U_{n} U_{n}^{H}$ represent respectively the signal part and the noise part, where Us is the signal subspace corresponding to $\mathrm{Vs}$ and $\mathrm{Un}$ is the noise subspace with the noise variance $\sigma^{2}$. The expression of normalized MUSIC spectrum is given by:

$$
\operatorname{Pmusic}(\theta)=\frac{a(\theta)^{H} a(\theta)}{a(\theta)^{H} U_{n} U_{n}^{H} a(\theta)}
$$

In literature, this expression could be written as:

$$
\operatorname{Pmusic}(\theta)=\frac{1}{a(\theta)^{H} U_{n} U_{n}^{H} a(\theta)}
$$

\subsection{Root-MUSIC}

Root-MUSIC is considered as one of many conversion of MUSIC algorithm, it is based on setting up the roots of a polynomial, this technique aims to decrease the computational complexity and improve its performance. Nevertheless, it is applicable only for a linear array spaced uniformly [17].

\subsection{ESPRIT algorithm}

Estimation of Signal Parameters via Rotational Invariance Technique (ESPRIT), is a subspace technique, used to estimate the angle of arrival (AOA) by determining the rotational operator $\Phi$ [21]. ESPRIT algorithm shows same performance as MUSIC algorithm with slight adjustment. The aims of ESPRIT strategy is to misuse the rotational invariance in the flag subspace, which is made by two clusters with a translational invariance structure [22]. ESPRIT algorithm have several advantages presented in the simplicity of Implementation, producing direction of arrival directly avoiding the search procedure, does not need much computation or storage requirement. Considering a linear array with two doublets and four elements, the two subarrays are received by [21].

$$
\begin{aligned}
& x_{1}(t)=A * S(t)+n_{1}(t) \\
& x_{2}(t)=A * \Lambda * S(t)+n_{2}(t)
\end{aligned}
$$

where

$$
\Lambda=\operatorname{diag}\left\{e^{j \varphi 1}, e^{j \varphi 2}, \ldots, e^{j \varphi D}\right\}
$$




$$
\begin{aligned}
& \varphi_{i}=-2 k d \sin (\theta) \\
& \lambda_{i}=e^{j \varphi_{i}}
\end{aligned}
$$

Where $\mathrm{D}<\mathrm{M}$ (number of signal hitting the subarrays). The two matrix $\mathrm{V}_{1}$ and $\mathrm{V}_{2}$ are creating by two subarrays, in which the eigenvectors are related by a unique nonsingular transformation matrix $\phi$ with a unique nonsingular transformation matrix $\mathrm{T}$.

$$
\begin{aligned}
& V_{2}=\phi V_{1} \\
& V_{1}=A T \\
& V_{2}=A \wedge T
\end{aligned}
$$

Than

$$
\wedge=\mathrm{T} \phi T^{-1}
$$

The eigenvalues of $\phi(\lambda i)$ must be equal to the diagonal elements of $\Lambda$. Once the eigenvalues are calculated the estimation of the angle of arrival can be made by:

$$
\theta_{i}=\sin ^{-1}\left(\frac{\arg \left(\lambda_{i}\right)}{k d}\right)
$$

\subsection{WSF algorithm}

Weighted Subspace Fitting (WSF) algorithm is an asymptotically proficient parametric method used for estimating the heights of different scatterers in the same azimuth-range resolution cell [23, 24]. Like the MUSIC and ESPRIT algorithms, the WSF algorithm represents a unified approach that also stand in needs of the knowledge of the number of directional sources, and the use of the decomposition technique for eigenvalues. the strongest eigenvectors in a diagonal matrix $\left(\hat{V}_{S}\right)$ and the corresponding eigenvectors in the signal subspace matrix $\left(\widehat{U}_{S}\right)[25]$ are used to accomplish this approach. The expression of WSF algorithm can be written as:

$$
\widehat{\theta} w s f=\operatorname{argmax}\left(\operatorname{Tr}\left(\Pi_{a}(\theta) \widehat{U}_{S} W \widehat{U}_{S}^{H}\right)\right)
$$

$\Pi_{a}(\theta)$ represente The projection matrix onto the column space of $\mathrm{a}(\theta)$, and $\mathrm{W}$ is a weighting matrix to reduce the impact of the subspace swap [26].

\subsection{Root-WSF algorithm}

Root-WSF is the rooting version of Weighted Subspace Fitting, also referred as MODE technique. The aims of this method is to minimize the cost function with [27].

$$
f_{M O D E}(\theta)=\operatorname{Tr}\left(P_{a(\theta)}^{\perp} \widehat{U}_{S} W_{M O D E} \widehat{U}_{S}^{H}\right)
$$

Where:

$$
\begin{aligned}
& P_{a(\theta)}^{\perp}=I_{M}-a(\theta)\left(a(\theta)^{H} a(\theta)\right)^{-1} a(\theta)^{H} \\
& W_{M O D E}=\left(\hat{V}_{S}-\hat{\sigma}^{2} I\right) \hat{V}_{S}^{-1} \\
& \hat{\sigma}^{2}=\frac{1}{N-M} \operatorname{Tr}\left(\hat{V}_{n}\right)
\end{aligned}
$$

$W_{M O D E}$ correspond to the asymptotic-optimum weight matrix, $\sigma^{2}$ is the noise variance and $P_{a(\theta)}^{\perp}$ indicate the orthogonal projection matrix of the array steering matrix [24, 28]. 


\section{RESULTS AND ANALYSIS}

In order to evaluate the performances of the different direction of arrival algorithms, three scenarios have been made, based on the changing parameters of the number of signals, the number of samples and the number of array elements. For this reason we consider a uniform linear array (ULA) of M the number of elementary antennas with the inter-element spacing of $\lambda / 2$, where $\lambda$ is the wavelength of incident radiation, $\mathrm{K}$ represents the number of samples or snapshots and the angles of arrival is given by AOA. All the sources are considered uncorrelated with the same frequency of $1 \mathrm{GHz}$ and with identical power. These simulations presented in this section are performed with MATLAB and SIMULINK R2015a.

\subsection{Result of a spatial spectrum}

In this part, we will evaluate the DOA algorithms based on the spatial spectrum study. For that, we proposed a system. The parameters of the proposed system can see in Table 1. The results of these simulations are presented as Figure 2, Figure 3 and Figure 4.

Table 1. Parameters of the proposed system

\begin{tabular}{cc}
\hline Parameters & Value \\
\hline Antenna & ULA \\
Number of the elements (M) & 10 \\
Spacing between elements (D) & $\lambda / 2$ \\
Received signals (S) & 3 \\
Angles of arrival (AOA) & $\Theta_{1}=-30^{\circ} ; \Theta_{2}=0^{\circ} ; \Theta_{3}=20^{\circ}$ \\
Number of samples (K) & 1024 \\
\hline
\end{tabular}

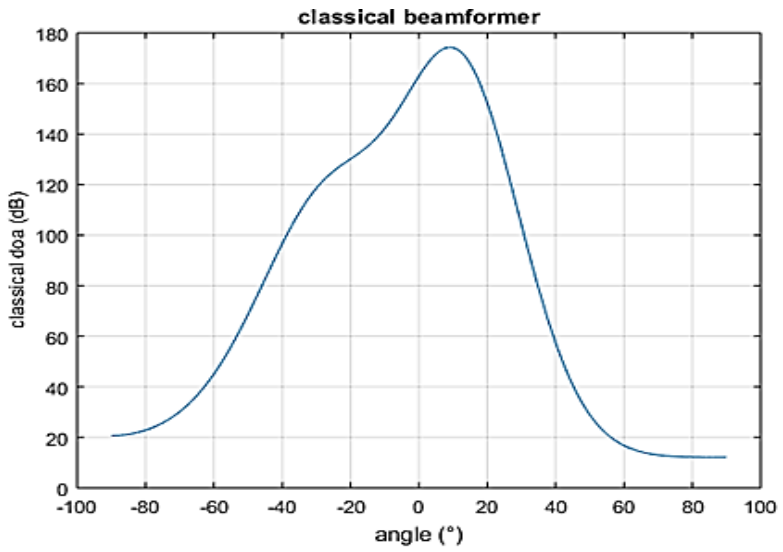

Figure 2. Conventional beamforming

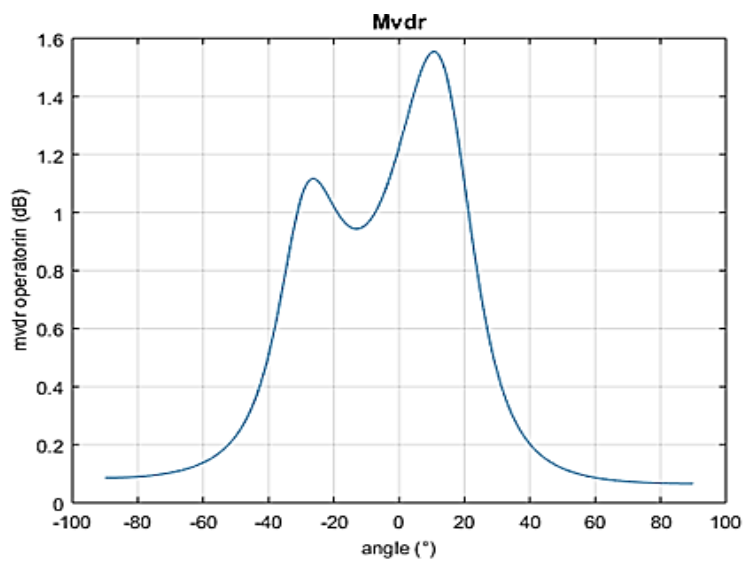

Figure 3. MVDR algorithm

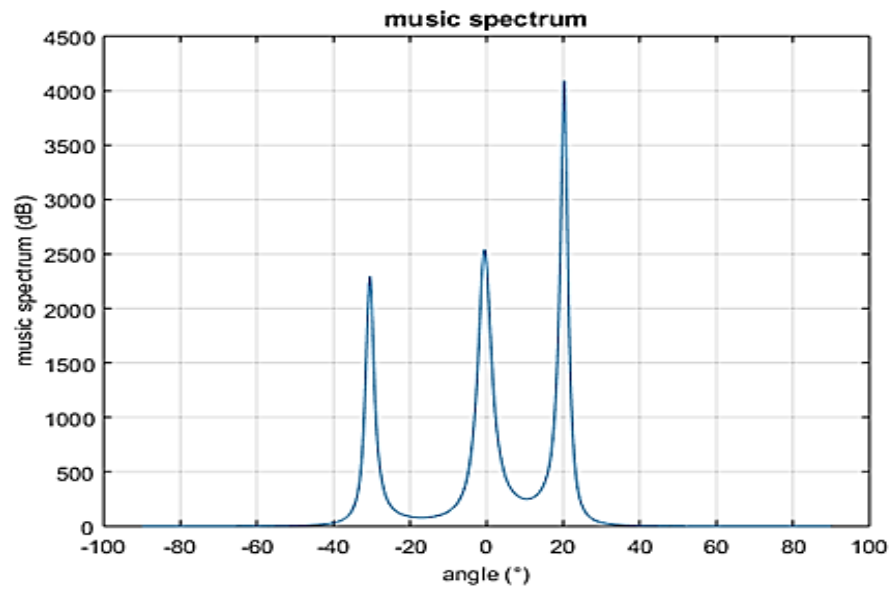

Figure 4. MUSIC algorithm 
The results obtained show three different algorithms. Figure 2 corresponds to the classic Beamforming giving unreliable results, where the proposed arrival angles are not detectable. Figure 3 indicates that the MVDR algorithm provides an acceptable result compared to the conventional algorithm. However, the angle of arrival of $\Theta_{2}=0^{\circ}$ is not conceivable. Figure 4 represents the MUSIC algorithm which gives the sharpest and clearest result among the three AOAs. Therefore, we can certainly conclude that the MUSIC algorithm stipulates the best result.

\subsection{DOA comparison}

In this section, we will analyze the performances of the different DOA algorithms, in order to locate the arrival angles and extract the best method. The proposed system remains the same as shown in Table 1 . The Table 2 presents this study. Table 2 is consistent with the study found in the spatial spectrum method, in which the MUSIC algorithm proves its effectiveness. we can also see that other algorithms like ROOTMUSIC and ROOT-WSF have distinguished themselves by giving good results.

Table 2. Comparative study of different DOA algorithms

\begin{tabular}{cccc}
\hline Type of DOA & $-30^{\circ}$ & AOA & $20^{\circ}$ \\
\hline MVDR & $-31^{\circ}$ & $0^{\circ}$ & $20^{\circ}$ \\
Beamscan & $-31^{\circ}$ & $1^{\circ}$ & $21^{\circ}$ \\
Beamspace ESPRIT & $-38.92^{\circ}$ & $-0.0009^{\circ}$ & $22.95^{\circ}$ \\
ESPRIT & $-42.29^{\circ}$ & $0^{\circ}$ & $18.66^{\circ}$ \\
MUSIC & $-30^{\circ}$ & $0^{\circ}$ & $20^{\circ}$ \\
ROOT-MUSIC & $-30.53^{\circ}$ & $-0.0009^{\circ}$ & $20.57^{\circ}$ \\
ROOT-WSF & $-30^{\circ}$ & $-0.0005^{\circ}$ & $20^{\circ}$ \\
\hline
\end{tabular}

\subsubsection{First scenario: Varying the number of elements}

After analyzing each DOA estimation algorithm, we will evaluate their performance according to certain criteria. First by changing the number of elementery array M from 6 to 8 then 10. Table 3 gives the results corresponding to two numbers of elementary antennas of 6 and 8 . While the number 10 is given by Table 2 where the DOA comparison study was established. We can notice that even by changing the number of antennas, the best results are only provided by ROOT-MUSIC and ROOT-WSF. As a result, the higher the number of antennas, the more accurate the results will be.

Table 3. The variation of the number of antenna elements for different DOA algorithms

\begin{tabular}{|c|c|c|c|c|c|c|}
\hline \multirow[t]{2}{*}{ Type of DOA } & \multicolumn{3}{|c|}{ AOA for $\mathrm{N}=6$} & \multicolumn{3}{|c|}{ AOA for $\mathrm{N}=8$} \\
\hline & $-30^{\circ}$ & $0^{\circ}$ & $20^{\circ}$ & $-30^{\circ}$ & $0^{\circ}$ & $20^{\circ}$ \\
\hline MVDR & $-30^{\circ}$ & $0^{\circ}$ & $18^{\circ}$ & $-27^{\circ}$ & $0^{\circ}$ & $20^{\circ}$ \\
\hline Beamscan & $-30^{\circ}$ & $-3^{\circ}$ & $20^{\circ}$ & $-28^{\circ}$ & $-2^{\circ}$ & $19^{\circ}$ \\
\hline Beamspace ESPRIT & $-32.03^{\circ}$ & $-0.0007^{\circ}$ & $20.04^{\circ}$ & $-37.36^{\circ}$ & $-0.0004^{\circ}$ & $27.16^{\circ}$ \\
\hline ESPRIT & $-33.39^{\circ}$ & $-0.0008^{\circ}$ & $22.35^{\circ}$ & $-29.47^{\circ}$ & $-0.0003^{\circ}$ & $25.08^{\circ}$ \\
\hline MUSIC & $-26.4^{\circ}$ & $-0.004^{\circ}$ & $11.2^{\circ}$ & $-30^{\circ}$ & $-1.2^{\circ}$ & $20^{\circ}$ \\
\hline ROOT-MUSIC & $-29.99^{\circ}$ & $-0.001^{\circ}$ & $20.1^{\circ}$ & $-25.4^{\circ}$ & $-0.0015^{\circ}$ & $21.56^{\circ}$ \\
\hline ROOT-WSF & $-30^{\circ}$ & $-0.0008^{\circ}$ & $20^{\circ}$ & $-30^{\circ}$ & $-0.001^{\circ}$ & $20^{\circ}$ \\
\hline
\end{tabular}

\subsubsection{2nd scenario: Varying the number of samples}

The Table 4 represents the varying number of samples from 100, 500, and 1024, applied in different DOA algorithms. The table corresponding to $\mathrm{K}=1024$ is the one with the comparison study as shown in Table 2. Like the previous table, the results show that the two algorithms ROOT-MUSIC and ROOT-WSF provide the best results. The ROOT-WSF algorithm gives the most accurate angles of arrival for the three proposed samples. It is also true that the larger the number of samples, the more precise it becomes.

\subsubsection{3rd scenario: Varying the number of signals}

We will now study the impact of the number of signals on the DOA estimate. The chosen numbers are 2 and 3. From Table 5, we can notice that two signals only need two angles of arrival to function, which is obvious, what is important to note is that for each of the DOA algorithms, we obtain the same result, corresponding to the angles of arrival that we seek. However, when the system switches to three signals, the only algorithms that enable the corresponding detection are ROOT-MUSIC and ROOT-WSF. 
Table 4. The variation of the number of samples for different DOA algorithms

\begin{tabular}{|c|c|c|c|c|c|c|}
\hline \multirow{2}{*}{ Type of DOA } & \multicolumn{3}{|c|}{ AOA for $K=100$} & \multicolumn{3}{|c|}{ AOA for $\mathrm{K}=500$} \\
\hline & $-30^{\circ}$ & $0^{\circ}$ & $20^{\circ}$ & $-30^{\circ}$ & $0^{\circ}$ & $20^{\circ}$ \\
\hline MVDR & $-31^{\circ}$ & $0^{\circ}$ & $20^{\circ}$ & $-30^{\circ}$ & $0^{\circ}$ & $20^{\circ}$ \\
\hline Beamscan & $-31^{\circ}$ & $1^{\circ}$ & $20^{\circ}$ & $-31^{\circ}$ & $1^{\circ}$ & $20^{\circ}$ \\
\hline Beamspace ESPRIT & $-30^{\circ}$ & $-8^{\circ}$ & $15.72^{\circ}$ & $-34.97^{\circ}$ & $-0.004^{\circ}$ & $18.27^{\circ}$ \\
\hline ESPRIT & $-29.29^{\circ}$ & $-0.0001^{\circ}$ & $13.86^{\circ}$ & $-35^{\circ}$ & $-0.004^{\circ}$ & $20.51^{\circ}$ \\
\hline MUSIC & $-30^{\circ}$ & $2.7^{\circ}$ & $20^{\circ}$ & $-30^{\circ}$ & $0.6^{\circ}$ & $19.6^{\circ}$ \\
\hline ROOT-MUSIC & $-31.01^{\circ}$ & $-0032^{\circ}$ & $20.29^{\circ}$ & $-30.32^{\circ}$ & $-0.0007^{\circ}$ & $19.71^{\circ}$ \\
\hline ROOT-WSF & $-30^{\circ}$ & $-0.002^{\circ}$ & $20^{\circ}$ & $-30^{\circ}$ & $-0.0002^{\circ}$ & $20^{\circ}$ \\
\hline
\end{tabular}

Table 5. The variation of the number of signals for different DOA algorithms

\begin{tabular}{cccccc}
\hline Type of DOA & \multicolumn{2}{c}{ AOA for S $=2$} & \multicolumn{3}{c}{ AOA for S $=3$} \\
& $-30^{\circ}$ & $20^{\circ}$ & $-30^{\circ}$ & $0^{\circ}$ & $20^{\circ}$ \\
\hline MVDR & $-30^{\circ}$ & $20^{\circ}$ & $-31^{\circ}$ & $0^{\circ}$ & $20^{\circ}$ \\
Beamscan & $-30^{\circ}$ & $20^{\circ}$ & $-31^{\circ}$ & $1^{\circ}$ & $21^{\circ}$ \\
Beamspace ESPRIT & $-30^{\circ}$ & $20^{\circ}$ & $-38.92^{\circ}$ & $-0.0009^{\circ}$ & $22.95^{\circ}$ \\
ESPRIT & $-30^{\circ}$ & $20^{\circ}$ & $-42.29^{\circ}$ & $0^{\circ}$ & $18.66^{\circ}$ \\
MUSIC & $-30^{\circ}$ & $20^{\circ}$ & $-30^{\circ}$ & $0^{\circ}$ & $20^{\circ}$ \\
ROOT-MUSIC & $-30^{\circ}$ & $20^{\circ}$ & $-30.53^{\circ}$ & $-0.0009^{\circ}$ & $20.57^{\circ}$ \\
ROOT-WSF & $-30^{\circ}$ & $20^{\circ}$ & $-30^{\circ}$ & $-0.0005^{\circ}$ & $20^{\circ}$ \\
\hline
\end{tabular}

\subsection{Comparaison with other studies}

Comparing our study with the related works previously published, we can notice that each of the proposed manuscripts [29-31] presents an incomplete study of the direction of arrival estimation algorithms. Therefore, we have tended in this article to complete these works by bringing together all the possibilities offered by the state of the art in order to apply them to new wireless technologies.

\subsection{Wi-Fi parameter}

For this study, we consider the same proposed system given in Table 2. Two Wi-Fi frequencies bands are used: $2.4 \mathrm{GHz}$ and $5 \mathrm{GHz}$. This analysis is performed only for the algorithms that gave good results found in the previous sections. The three DOA algorithms presented here have the same sensibility to the operating frequency, which is clearly remarkable in Table 6. Besides, the MUSIC algorithm cannot recognize the second angle of arrival at $0^{\circ}$. However, ROOT-WSF algorithm provided the best results with almost no error.

Table 6. The variation of the number of signals for different DOA algorithms

\begin{tabular}{ccccccc}
\hline Type of DOA & \multicolumn{3}{c}{$\mathrm{AOA}$ for $\mathrm{F}=2.4 \mathrm{GHz}$} & \multicolumn{3}{c}{ AOA for 5GHz } \\
& $-30^{\circ}$ & $0^{\circ}$ & $20^{\circ}$ & $-30^{\circ}$ & $0^{\circ}$ & $20^{\circ}$ \\
\hline MUSIC & $-24.4^{\circ}$ & undetectable & $13^{\circ}$ & $-21^{\circ}$ & undetectable & $6.7^{\circ}$ \\
ROOT-WSF & $-30^{\circ}$ & $0.0004^{\circ}$ & $20^{\circ}$ & $-30^{\circ}$ & $0.0009^{\circ}$ & $20^{\circ}$ \\
ROOT-MUSIC & $-33.62^{\circ}$ & $-0.0002^{\circ}$ & $20.88^{\circ}$ & $-30.78^{\circ}$ & $-0.00013^{\circ}$ & $20.04^{\circ}$ \\
\hline
\end{tabular}

\section{CONCLUSION}

The study established in this paper is based on a uniform linear array (ULA) of M antenna elements with the inter-element spacing of $\lambda / 2$, where all the sources are assumed uncorrelated. We first evaluated the impact of the array elements from 6 to 10, in which the ROOT-MUSIC and the ROOT-WSF algorithms proved their effectiveness. Then we evaluate the impact of the number of sources on the DOA algorithms. The results clearly show that the increase in the number of sources leads to a certain complexity in the proposed DOA algorithms, in this case also, ROOT-MUSIC and the ROOT-WSF algorithms have achieved the best results. Finally, we evaluate the number of snapshots or (number of samples) that gives the identical results.

From this study, it can be seen that ROOT-WSF and ROOT-MUSIC algorithms have provided the best result on the different impacts that we have worked with, therefore, these two techniques will be the most feasible in the upcoming research. As for the operating frequency, the two $2.4 \mathrm{GHz}$ and $5 \mathrm{GHz} \mathrm{Wi}-\mathrm{Fi}$ bands gave the best result with the ROOT-WSF algorithm in comparison with the MUSIC and ROOTMUSIC algorithm. In terms of accuracy, the ROOT-WSF algorithm provides the most accurate detection of the angles of arrival in the three proposed scenarios with almost no error. This makes it the best choice for the implantation in the smart antenna system. 


\section{REFERENCES}

[1] M. Z. Chowdhury, et al., "A Comparative Survey of Optical Wireless Technologies: Architectures and Applications," IEEE Access, vol. 6, pp. 9819-9840, 2018.

[2] X. Huang, "A Hybrid Adaptive Antenna Array," IEEE Transactions on Wireless Communications, vol. 9, 2010.

[3] N. I. Smirnov, et al., "Increase in the efficiency of the coverage area in the cellular communication systems," Systems of Signal Synchronization, Generating and Processing in Telecommunications (SINKHROINFO), Kazan, pp. 1-4, 2017.

[4] L. C. Godara, "Applications of Antenna Arrays to Mobile Communications. Performance Improvement, Feasibility, and System Considerations," Proceedings of IEEE, vol. 85, pp. 1031-1060, 1997.

[5] J. R. Mohammed, "Comparative Performance Investigations of Stochastic and Genetic Algorithms Under Fast Dynamically Changing Environment in Smart Antennas," International Journal of Electrical and Computer Engineering (IJECE), vol. 2, pp. 98-105, 2012.

[6] Balasem S. S., et al., "Beam forming Algorithms Technique by Using MVDR and LCMV," International E-Conference on Information Technology and Applications (IECITA), 2012.

[7] S. S. Moghaddam, et al., "A novel array geometry to improve DOA estimation of narrowband sources at the angles close to the array end fire."

[8] J. Xu, et al., "Joint Range and Angle Estimation Using MIMO Radar With Frequency Diverse Array," IEEE Transactions on Signal Processing, vol. 63, pp. 3396-3410, 2015.

[9] W. Q. Wang, "Overview of frequency diverse array in radar $\mathrm{r}$ and navigation applications," IET Radar, Sonar \& Navigation, 2016.

[10] S. Ouelha, et al., "Improving DOA Estimation Algorithms Using High-Resolution Quadratic Time-Frequency Distributions," IEEE Transactions on Signal Processing, vol. 65, pp. 5179-5190, 2017.

[11] J. G. A. Olague, et al., "Efficient Adaptive Algorithms for DOA Estimation in Wireless Communications," International Journal of Communications, Network and System Sciences, vol. 3, 2010.

[12] K. A. Kumbar, "Adaptive Beamforming Smart Antenna for Wireless Communication System," International Research Journal of Engineering and Technology (IRJET), vol. 02, 2015.

[13] M. Devendra and K. Manjunathachari, "DOA estimation of a system using MUSIC method," International Conference on Signal Processing and Communication Engineering Systems, Guntur, pp. 309-313, 2015.

[14] Y. Zhao, et al., "Efficient sparse representation method for wideband DOA estimation using focusing operation," IET Radar, Sonar \& Navigation, vol. 11, pp. 1673-1678, 2017.

[15] S. Waghmare, et al., "Beamforming Smart Antenna LCMV \& MVDR Algorithm," International Journal of Advanced Research in Computer Engineering \& Technology (IJARCET), vol. 4, 2015.

[16] MATLAB, “The MathWorks, Direction of Arrival Estimation,” Inc, Natick, United States.

[17] B. Boustani, et al., "Performance analysis of Direction Of Arrival estimation under hard condition," 4th International Conference on Optimization and Applications (ICOA), Mohammedia, pp. 1-5, 2018.

[18] S. Lukose, "A Study on Various Types of Beamforming Algorithms," IJSTE, vol. 2, 2010.

[19] J. Capon, "High-resolution frequency-wave number spectral analysis," Proc. IEEE, vol. 57, pp. 1408-1518, 1969.

[20] N. Nemri, et al., "Synthesis and Implementation (In STM8S) of Phased Circular Antenna Arrays Using Taguchi Method," International Journal of Electrical and Computer Engineering (IJECE), 2016.

[21] MATLAB, "The MathWorks, Direction of Arrival Estimation with Beamscan, MVDR, and MUSIC," Inc, Natick, United States.

[22] E. T. Aung and S. S. Y. Mon, "Performance comparison of DOA estimation algorithms for smart antenna," IJECSE, vol. 3, pp. 167-174.

[23] T. B. Lavate, et al., "Performance Analysis of MUSIC and ESPRIT DOA Estimation Algorithms for Adaptive Array the Smart Antenna in Mobile Communication," International Journal of Computer Netwoks (IJCN), vol. 2.

[24] Viberg M., et al., "Detection and estimation in sensor arrays using weighted subspace fitting," IEEE Trans. Signal Process, vol. 39, pp. 2436-2449, 1991.

[25] Y. Huang and L. F. Famil, "Building Height Estimation Using Multibase Linel-Band Sardataand Polarimetric Weighted Subspace Fitting Methods," University of Rennes 1, Institute of Telecommunication and Electronics.

[26] "Direction of arrival estimation methods," CRC Press LLC, 2004.

[27] B. A. Johnson, et al., "The Role of Subspace Swap in Maximum Likelihood Estimation Performance Breakdown," IEEE Proc. of Int. Conf. on Acoustics, Speech, and Signal Processing, pp. 2469-2472, 2008.

[28] “Array and Statistical Signal Processing," Academic Press Library in Signal Processing. Academic Press, 2013.

[29] M. U. Shahid, et al., "Comparative analysis between direction of arrival algorithms," International Conference on Infocom Technologies and Unmanned Systems (Trends and Future Directions) (ICTUS), Dubai, pp. 451-454, 2017.

[30] N. A. Baig and M. B. Malik, "Comparison of Direction of Arrival (DOA) Estimation Techniques for Closely Spaced Targets," International Journal of Future Computer and Communication, vol. 2, 2013.

[31] Y. Khmou, et al., "Comparative Study between Several Directions of Arrival Estimation Methods," Journal of Telecommunications and Information Technology, National Institute of Telecommunications, Warsaw, vol. 1, pp. 41-48, 2014. 


\section{BIOGRAPHIES OF AUTHORS}
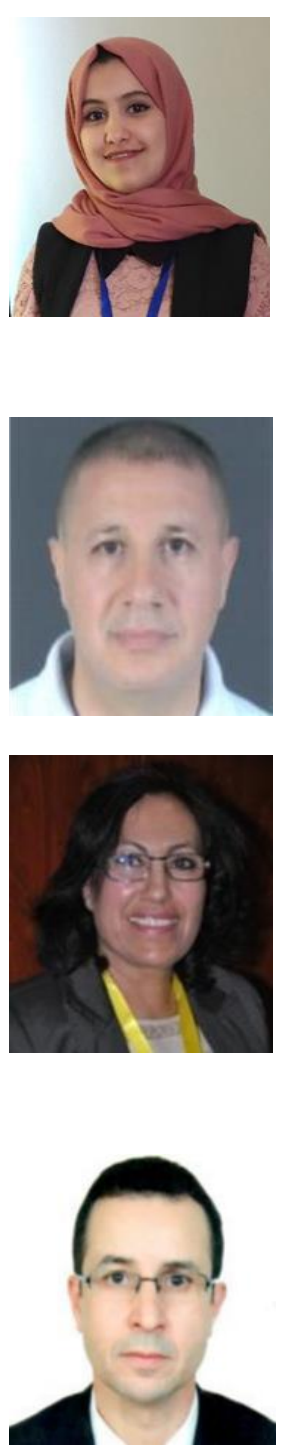

Btissam BOUSTANI born in Mohammedia, Morocco on June 24, 1989. In 2012 She had got her license degree in physical science for the engineer, at the University Hassan II Mohammedia Casablanca- Morocco (FSTM), then she had got a Master's degree in system and computer signals at Fez University of Sciences Dhar El Mahraz. She is currently a Ph.D. student in the Laboratory of Electronics, Energy, Automatics and Data Processing (EEA\&TI) Hassan II University, Mohammedia-Casablanca, Morocco. Her works studies and interests are focused on the optimization of the performance of smart antenna system to create a beamforming adjustable to the needs of the system improvements, assuring to locate and track the desired signal, under the supervision of Pr. A. Baghdad, Professor in Electrical engineering department at the same University.

Abdennaceur BAGHDAD is a holder of a doctorate in the Electronics in 1992 at the University of Lille - France. He is University Professor (PES) at the University Hassan II Mohammedia Casablanca- Morocco (FSTM) where he teaches the electronics, Hyperfrequencies, antenna and telecommunication. He is a member of the laboratory EEA\&TI (Electonics, Energy, Automatic and information Processing). The research works of A. Baghdad concerns the communication and Information Technology (Electronics Systems and Telecommunication). He supervises doctoral theses and he is a co-author of several national and international publications. He was a member of steering committees of three international congresses in the same domain of research.

Aïcha SAHEL is a holder of a doctorate in Electronics and Image Processing in 1996 at the University of Poitiers - France. She is university Professor at the University Hassan II Mohammedia - Casablanca - Morocco (FSTM) where she teaches electronics, signal processing, image processing and Telecommunication. She is a member of the laboratory EEA\&TI (Electronics, Electrotechnics, Automatic and Information processing). The research works of A. SAHEL concern the Communication and Information Technology (Electronics Systems, Signal/Image Processing and Telecommunication). She co-supervises doctoral theses and she is a co-author of several national and international publications. She is a member of financed research projects. She was a member of steering committees of three international congresses in the same domain of research.

Abdelmajid BADRI is a holder of a doctorate in Electronics and Image Processing in 1992 at the University of Poitiers - France. In 1996, he obtained the diploma of the authorization to Manage Researches (HDR) to the University of Poitiers - France, on the image processing. He was a University Professor (PES-C) at the University Hassan II Mohammedia-Casablanca Morocco (FSTM). In 2018, he becames director of the superior school of technology of Casablanca Morocco (EST). He is a member of the laboratory EEA\&TI (Electonics, Energy, Automatic and information Processing) which he managed since 1996. The research works of A. Badri concerns the communication and Information Technology (Electronics Systems, Signal/Image Processing and Telecommunication). He is qualified by CNU-France in 61st section: informatics engineering, automatic and signal processing. He managed several doctoral theses. He is a co-author of several national and international publications. He is responsible for several research projects financed by the ministry or by the industrialists. He was member of several committees of programs of international conferences and president of three international congresses in the same domain. He is a member and responsible in several scientific associations in touch with his domain of research. 